no. ITL-96-7

c. 2

US Army Corps of Engineers

Waterways Experiment Station

US-CE-C Property of the

United States Government

\title{
A Hardware Analysis of the Fundamental Iterative Algorithm for Decoding A $(17,9)$ Binary BCH Code
}

by Roy L. Campbell, Jr.

Approved For Public Release; Distribution Is Unlimited

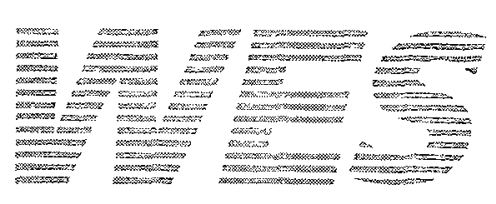

Research Library

US Army Engheer Waterways

Experiment Station

Vicksburg. Mississippi

Prepared for Headquarters, U.S. Army Corps of Engineers 
The contents of this report are not to be used for advertising, publication, or promotional purposes. Citation of trade names does not constitute an official endorsement or approval of the use of such commercial products. 
TA'T
W 34
$n 0 . \pm T L-96-7$
C.2

Technical Report ITL-96-7

August 1996

\section{A Hardware Analysis of the Fundamental Iterative Algorithm for Decoding A $(17,9)$ Binary BCH Code}

by Roy L. Campbell, Jr.

U.S. Army Corps of Engineers

Waterways Experiment Station

3909 Halls Ferry Road

Vicksburg, MS 39180-6199

Final report

Approved for public release; distribution is unlimited 


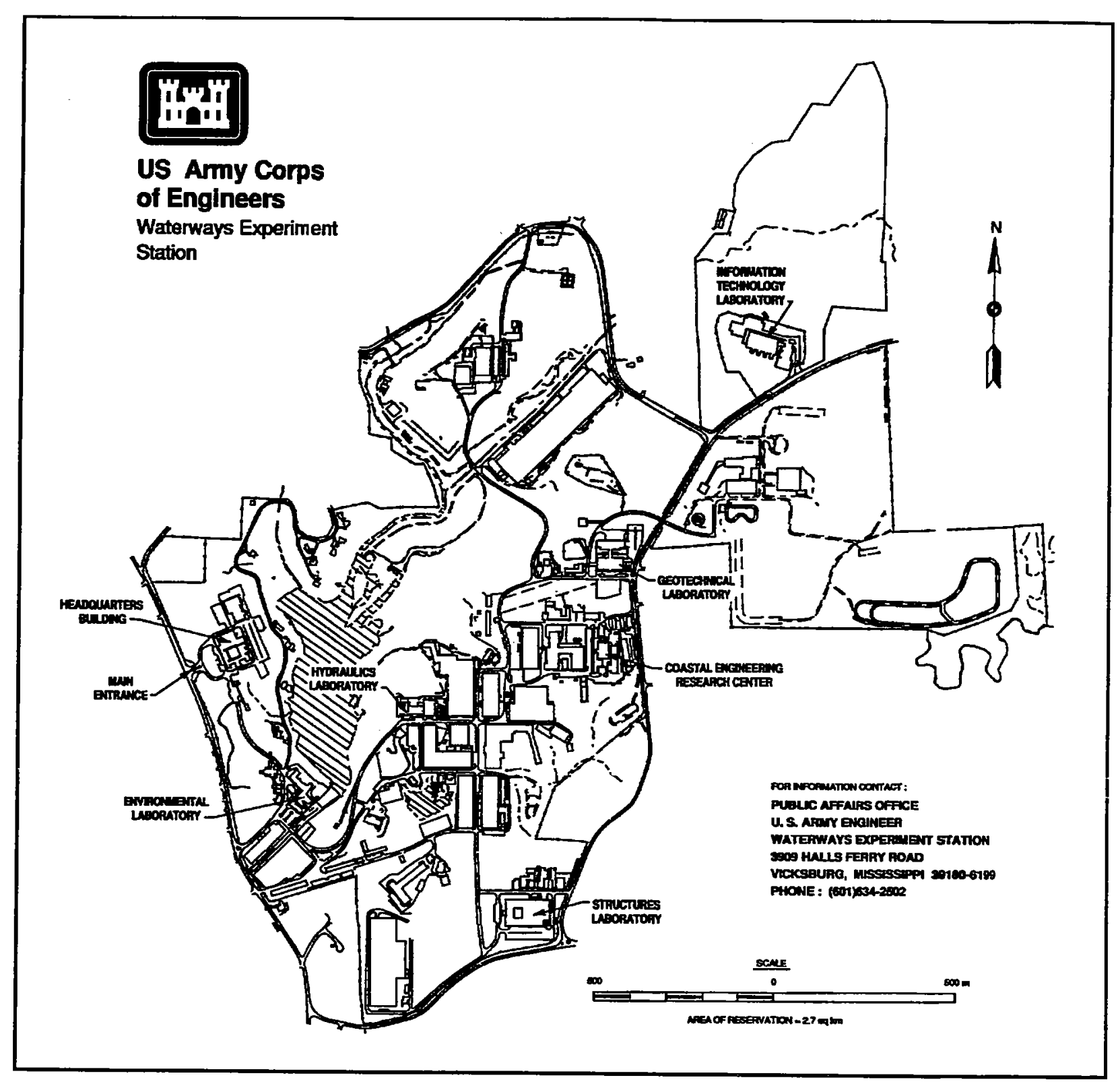

Waterways Experiment Station Cataloging-in-Publication Data

Campbell, Roy L.

A hardware analysis of the fundamental iterative algorithm for decoding a $(17,9)$ binary BCH code / by Roy L. Campbell ; prepared for U.S. Army Corps of Engineers.

44 p. : ill. ; $28 \mathrm{~cm}$.-- (Technical report ; ITL-96-7)

Includes bibliographic references.

1. Computer algorithms. 2. Algorithms. 3. Decoders (Electronics) I. United $\overrightarrow{\text { States. }}$ Army. Corps of Engineers. II. U.S. Army Engineer Waterways Experiment Station. III. Information Technology Laboratory (U.S. Army Engineer Waterways Experiment Station) IV. Title. V. Series: Technical report (U.S. Army Engineer Waterways Experiment Station) ; ITL-96-7.

TA7 W34 no.ITL-96-7 


\section{PREFACE}

This report was prepared by Roy L. Campbell, Jr., Information Management

Division (IMD), Information Technology Laboratory (ITL), U.S. Army Engineer

Waterways Experiment Station (WES), Vicksburg, Mississippi, as a thesis to the faculty of Mississippi State University in partial fulfillment of the requirements for the Degree of Master of Science in Electrical Engineering in May of 1996. This report was prepared under the supervision of Mr. Murray Huffman, Chief, IMD, and Dr. N. Radhakrishnan, Director, ITL.

At the time of publication of this report, Dr. Robert W. Whalin was Director of WES. COL Bruce K. Howard, EN, was Commander. 


\section{ACKNOWLEDGMENTS}

Sincere appreciation is extended to Dr. William J. Ebel for his guidance throughout this endeavor, to the Honda Corporation for its financial support of the preceding course work, and to Dr. N. Radhakrishnan, Director of the Information Technology Laboratory, Waterways Experiment Station, for his support and understanding during the thesis. 


\section{TABLE OF CONTENTS}

\section{Page}

ACKNOWLEDGMENTS $\ldots \ldots \ldots \ldots \ldots \ldots \ldots \ldots \ldots \ldots \ldots$ ii

LIST OF TABLES $\ldots \ldots \ldots \ldots \ldots \ldots \ldots \ldots \ldots \ldots \ldots \ldots$ iv

LIST OF FIGURES $\ldots \ldots \ldots \ldots \ldots \ldots \ldots \ldots \ldots \ldots \ldots \ldots \ldots$

CHAPTER

I. INTRODUCTION $\ldots \ldots \ldots \ldots \ldots \ldots \ldots \ldots \ldots \ldots \ldots \ldots$

II. DECODING ALGORITHMS $\ldots \ldots \ldots \ldots \ldots \ldots \ldots \ldots \ldots \ldots \ldots$

The Berlekamp-Massey Algorithm $\ldots \ldots \ldots \ldots \ldots \ldots \ldots \ldots \ldots$

The Fundamental Iterative Algorithm . . . . . . . . . . . . 7

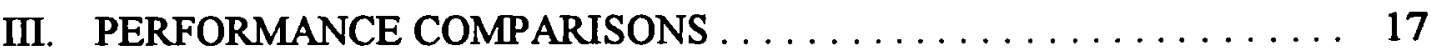

The Simulated System $\ldots \ldots \ldots \ldots \ldots \ldots \ldots \ldots \ldots \ldots$

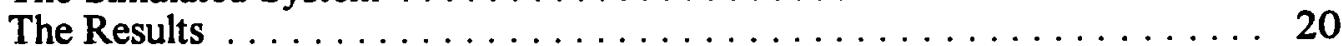

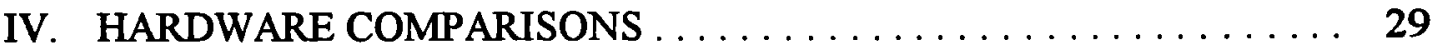

Critical Path for the BMA . . . . . . . . . . . . . . . . 29

Critical Path for the FIA . . . . . . . . . . . . . . . 30

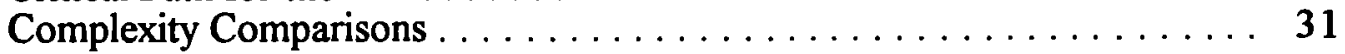

V. SUMMARY AND CONCLUSIONS $\ldots \ldots \ldots \ldots \ldots \ldots \ldots$

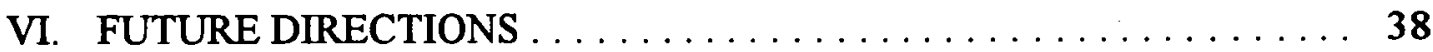

BIBLIOGRAPHY $\ldots \ldots \ldots \ldots \ldots \ldots \ldots \ldots \ldots \ldots \ldots \ldots \ldots \ldots \ldots$ 


\section{LIST OF TABLES}

Table

Page

4.1 HARDWARE COMPLEXITY COMPARISON $\ldots \ldots \ldots \ldots \ldots \ldots, 31$ 


\section{LIST OF FIGURES}

Figure Page

3.1 THEORETICAL PERFORMANCE CURVES FOR $(17,9)$ BCH CODE . 23

3.2 BMA STATISTICAL TEST WITH DECODING ERRORS DISABLED FOR $(17,9)$ BCH CODE $\ldots \ldots \ldots \ldots \ldots \ldots \ldots \ldots \ldots \ldots \ldots \ldots \ldots \ldots \ldots \ldots, 24$

3.3 FIA STATISTICAL TEST WITH DECODING ERRORS DISABLED FOR $(17,9)$ BCH CODE $\ldots \ldots \ldots \ldots \ldots \ldots \ldots \ldots \ldots \ldots \ldots \ldots \ldots \ldots \ldots, 25$

3.4 BMA STATISTICAL TEST WITH NO RESTRICTIONS FOR $(17,9)$ BCH CODE $\ldots \ldots \ldots \ldots \ldots \ldots \ldots \ldots \ldots \ldots \ldots \ldots \ldots \ldots \ldots, 26$

3.5 FIA STATISTICAL TEST WITH NO RESTRICTIONS FOR $(17,9)$ BCH CODE $\ldots \ldots \ldots \ldots \ldots \ldots \ldots \ldots \ldots \ldots \ldots \ldots, 27$

3.6 SIMULATION PERFORMANCE CURVES FOR $(17,9)$ BCH CODE .. 28

4.1 BMA HARDWARE LAYOUT FOR A $(17,9)$ BCH DECODER . . . . . 32

4.2 FIA HARDWARE LAYOUT FOR A $(17,9)$ BCH DECODER ...... 33 


\section{CHAPTER I}

\section{INTRODUCTION}

A $\mathrm{BCH}$ code is a linear cyclic block code with a generator polynomial, $g(x)$, that is a product of minimal polynomials, where the $2 t$ designed zeros of $g(x)$ have contiguous powers of $\beta$. Note that $t$ is the designed number of correctable errors, $d_{o}=2 t+1$ is the designed distance, $\beta=\alpha^{b}, \alpha$ is a primitive element of the field $G F(Q), b=(Q-1) / n$, and $n$ is the length of a channel codeword. Often the true minimum distance of the $\mathrm{BCH}$ code is larger than $d_{o}$. A decoder based on the Berlekamp-Massey Algorithm (BMA) makes use of the designed distance only [1], however the Fundamental Iterative Algorithm (FIA) can be used to exploit some or all of the code's unused distance, in many cases increasing the number of correctable errors [3].

Often, the critical issue is not the number of correctable errors, but the performance gain/hardware complexity ratio. Since hardware complexity is difficult to measure, this thesis describes and compares hardware realizations for both decoding algorithms in light of their corresponding performance gains. 


\section{CHAPTER II \\ DECODING ALGORITHMS}

\section{The Berlekamp-Massey Algorithm}

Given a BCH code over $G F(q)$ with a $G F\left(q^{m}\right)$ error locator field, the generator polynomial is $g(x)=L C M\left[m_{1}(x), m_{2}(x), \ldots, m_{2 t}(x)\right]$, where $m_{i}(x)$ is the minimal polynomial for $\beta^{j_{o}+i-1}$, for $i=1,2, \ldots, 2 t$. Note that $0 \leq j_{o} \leq q^{m}-2, \beta=\alpha^{b}, \alpha$ is a primitive element of $G F\left(q^{m}\right), b=\left(q^{m}-1\right) / n$, and $n$ is the number of code symbols in a codeword. Considering the $k$ coefficients of $i(x)$ as an information block, a channel codeword is obtained by polynomial multiplication

$$
c(x)=i(x) g(x)
$$

where $\operatorname{deg}\{i(x)\}=k-1, \operatorname{deg}\{g(x)\}=n-k, \operatorname{deg}\{c(x)\}=n-1$, and $k<n$.

The channel errors can be expressed as

$$
e(x)=\sum_{j=1}^{v} e_{l_{j}} \cdot x^{l_{j}}
$$

where $\nu$ is the number of errors, $l_{j}$ is the $j^{\text {th }}$ error position, and $e_{l_{j}}$ is the corresponding error weight. Assuming the $n$ symbols of the received codeword are the coefficients of $v(x)$,

$$
v(x)=c(x)+e(x)
$$


since the channel errors are modeled to be additive. The syndrome values can be calculated as

$$
S_{i}=v\left(\beta^{j_{0}+i-1}\right)=c\left(\beta^{j_{0}+i-1}\right)+e\left(\beta^{j_{0}+i-1}\right)=e\left(\beta^{j_{0}+i-1}\right),
$$

for $i=1,2, \ldots, 2 t$. By defining $Y_{j}=e_{l_{j}}$ and $X_{j}=\beta^{l_{j}}$ for $j=1,2, \ldots, \nu$, the syndrome equation can be rewritten as

$$
S_{i}=\sum_{j=1}^{v} Y_{j} \cdot X_{j}^{\left(j_{o}+i-1\right)}
$$

for $i=1,2, \ldots, 2 t$. Therefore, there are $2 t$ non-linear simultaneous equations that relate the channel errors to the syndromes. An error locator polynomial has roots $X_{j}^{-1}$, for $j=1,2, \ldots, \nu$, and can be used with a little deduction to reveal the errorsyndrome relationship

$$
\begin{gathered}
\Lambda(x)=\Lambda_{\nu} \cdot x^{\nu}+\Lambda_{\nu-1} \cdot x^{\nu-1}+\ldots+\Lambda_{1} \cdot x+1=\left(1-x \cdot X_{1}\right)\left(1-x \cdot X_{2}\right) \ldots\left(1-x \cdot X_{\nu}\right), \\
Y_{j} X_{j}^{i+\nu} \cdot \Lambda\left(X_{j}^{-1}\right)=Y_{j} X_{j}^{i+\nu} \cdot\left(\Lambda_{\nu} \cdot X_{j}^{-\nu}+\Lambda_{\nu-1} \cdot X_{j}^{-\nu+1}+\ldots+\Lambda_{1} \cdot X_{j}^{-1}+1\right)=0 \\
Y_{j} \cdot\left(\Lambda_{\nu} \cdot X_{j}^{i}+\Lambda_{\nu-1} \cdot X_{j}^{i+1}+\ldots+\Lambda_{1} \cdot X_{j}^{\nu+i-1}+X_{j}^{\nu+i}\right)=0 \\
\sum_{j=1}^{\nu} Y_{j} \cdot\left(\Lambda_{\nu} \cdot X_{j}^{i}+\Lambda_{\nu-1} \cdot X_{j}^{i+1}+\ldots+\Lambda_{1} \cdot X_{j}^{\nu+i-1}+X_{j}^{\nu+i}\right)=0 \\
\Lambda_{\nu} \sum_{j=1}^{\nu} Y_{j} X_{j}^{i}+\Lambda_{\nu-1} \sum_{j=1}^{\nu} Y_{j} X_{j}^{i+1}+\ldots+\Lambda_{1} \sum_{j=1}^{\nu} Y_{j} X_{j}^{\nu+i-1}+\sum_{j=1}^{\nu} Y_{j} X_{j}^{\nu+i}=0 .
\end{gathered}
$$

Assuming $j_{o}=1$, 


$$
\begin{gathered}
\Lambda_{\nu} \cdot S_{i}+\Lambda_{\nu-1} \cdot S_{i+1}+\ldots+\Lambda_{1} \cdot S_{\nu+i-1}+S_{\nu+i}=0 \\
\Lambda_{1} \cdot S_{\nu+i-1}+\ldots+\Lambda_{\nu-1} \cdot S_{i+1}+\Lambda_{\nu} \cdot S_{i}=-S_{\nu+i}
\end{gathered}
$$

for $i=1,2, \ldots, 2 t$. All $2 t$ equations can be expressed at once with the following matrix equation

$$
\left[\begin{array}{ccccc}
S_{1} & S_{2} & \ldots & S_{\nu-1} & S_{\nu} \\
S_{2} & S_{3} & \ldots & S_{\nu} & S_{\nu+1} \\
\cdot & & & & \cdot \\
\cdot & & & & \cdot \\
S_{\nu} & S_{\nu+1} & \ldots & S_{2 \nu-2} & S_{2 \nu-1}
\end{array}\right] \cdot\left[\begin{array}{c}
\Lambda_{\nu} \\
\Lambda_{\nu-1} \\
\cdot \\
\cdot \\
\Lambda_{1}
\end{array}\right]=\left[\begin{array}{c}
-S_{\nu+1} \\
-S_{\nu+2} \\
\cdot \\
\cdot \\
-S_{2 \nu}
\end{array}\right]
$$

Assuming that $\nu \leq t$, the BMA can be used to find the coefficients of the error locator polynomial $\Lambda(x)[1]$. The initialization of the BMA depends on the first syndrome. If $S_{1} \neq 0, \Lambda^{(0)}(x)=1, \Lambda^{(1)}(x)=1-S_{1} x, L=1, m=1, \Delta m=S_{1}$, and $r=2$; otherwise, $\Lambda^{(-1)}(x)=\Lambda^{(0)}(x)=\Lambda^{(1)}(x)=1, L=0, m=0, \Delta m=1$, and $r=2 . \Lambda^{(r)}(x)$ is the current approximation of the error locator polynomial, $L$ is the stored register length, $m$ is the stored stage number, $\Delta m$ is the stored syndrome error, $r$ is the current stage number, $S_{r}^{\prime}$ is the approximation of the $r^{\text {th }}$ syndrome, and $\Delta r$ is the current syndrome error. The following pseudo code describes the inductive loop of the BMA: 


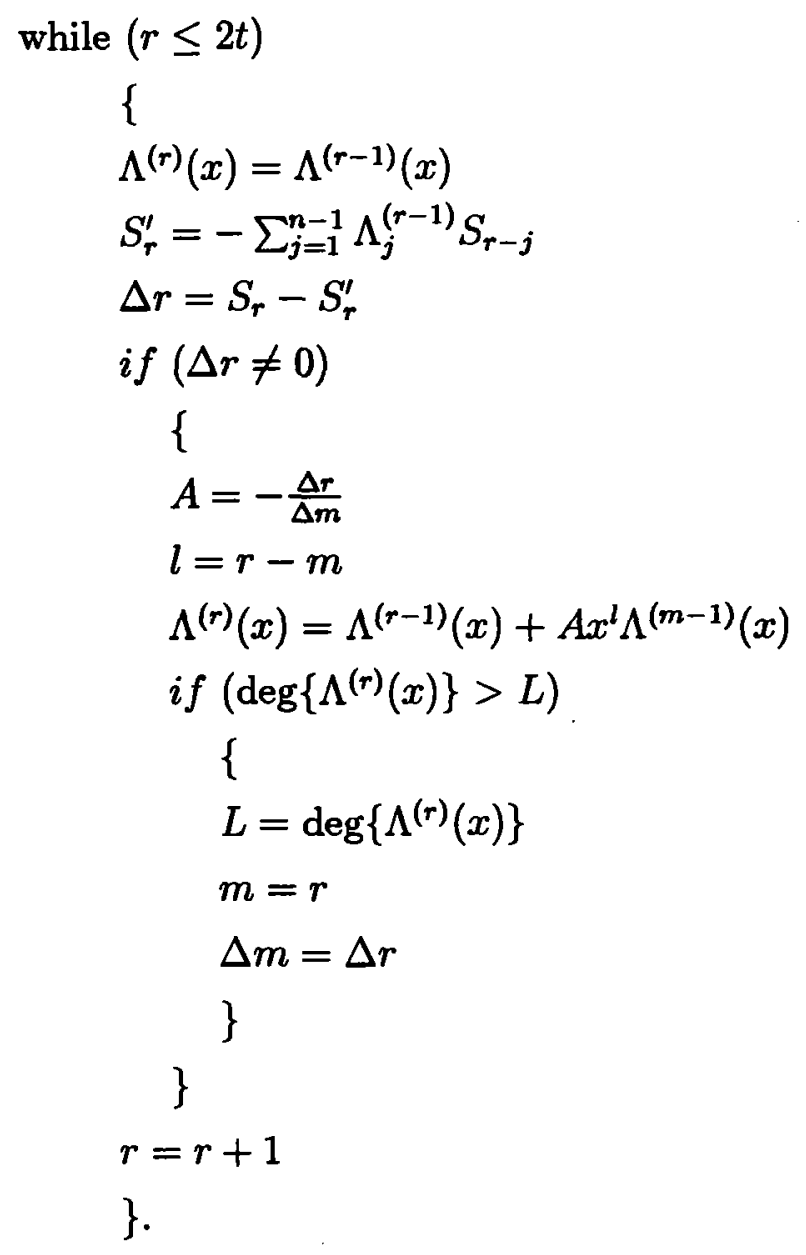

$\Lambda^{(2 t)}(x)$ is the error locator polynomial $\Lambda(x)$, and $L$ is the number of received symbol errors $\nu$. If $\operatorname{deg}\{\Lambda(x)\} \neq L$, a decoding failure has occurred [1].

A Chien search is an evaluation of a polynomial at all possible values of $\beta^{x}$, where $x$ is arbitrary. This search can be used to find the $\nu$ zeros of $\Lambda(x)$. The Forney Algorithm [1] can then be used to find the error weights

$$
Y_{j}=-\frac{\Omega\left(\beta^{-l_{j}}\right)}{\Lambda^{\prime}\left(\beta^{-l_{j}}\right)},
$$


where

$$
\begin{aligned}
\Omega(x) & =S(x) \Lambda(x)\left(\bmod x^{2 t}\right) \\
S(x) & =\sum_{j=1}^{2 t} S_{j} x^{(j-1)} \\
\Lambda^{\prime}(x) & =\sum_{i=1}^{\nu}\left[\sum_{j=1}^{i} 1\right] \Lambda_{i} x^{(i-1)}
\end{aligned}
$$

Now, $e(x)$ is fully specified and

$$
\begin{aligned}
& c(x)=v(x)-e(x), \\
& i(x)=c(x) / g(x) .
\end{aligned}
$$

For the $(17,9) \mathrm{BCH}$ code, the error locator field is $G F\left(2^{8}\right), b=15, \beta=\alpha^{15}$, $g(x)=(x-\beta) \cdot\left(x-\beta^{2}\right) \cdot\left(x-\beta^{4}\right) \cdot\left(x-\beta^{8}\right) \cdot\left(x-\beta^{9}\right) \cdot\left(x-\beta^{13}\right) \cdot\left(x-\beta^{15}\right) \cdot\left(x-\beta^{16}\right)$, and the syndromes are calculated as follows, assuming $j_{\circ}=1$,

$$
\begin{aligned}
& S_{1}=v(\beta) \\
& S_{2}=v\left(\beta^{2}\right)=\left(S_{1}\right)^{2} .
\end{aligned}
$$

This last equation can be proven by considering a generic polynomial defined over $G F(q)$, where $q$ is the characteristic of the field $G F(Q)$,

$$
f(x)=\sum_{i=0}^{n-1} f_{i} \cdot x^{i}
$$

If the argument of $f(x)$ is raised to the $q$ power, 


$$
f\left(x^{q}\right)=\sum_{i=0}^{n-1} f_{i} \cdot x^{i q}
$$

Since $f_{i} \in G F(q), f_{i}=f_{i}^{q}$. Therefore [1],

$$
f\left(x^{q}\right)=\sum_{i=0}^{n-1} f_{i}^{q} \cdot x^{i q}=\left[\sum_{i=0}^{n-1} f_{i} \cdot x^{i}\right]^{q}=[f(x)]^{q}
$$

For all single-error correcting binary $\mathrm{BCH}$ codes, the assumption that $j_{o}=1$ forces the Berlekamp-Massey Algorithm to degenerate into a mere comparison. If $S_{1}=0$, then $\Lambda(x)=1$ and $L=0$. Therefore, the received codeword has no errors, or a decoding error has occurred. In this case, the received codeword is passed as the decoded codeword. If $S_{1} \neq 0$, then $\Lambda(x)=1-S_{1} \cdot x$ and $L=1$. Therefore, the received codeword has a single error, or a decoding error has occurred. For this case, the zero of the error locator polynomial is extracted by simply noting that for $\Lambda(x)=0, x=1 / S_{1}$. Next, the error location is given by $l_{1}=\log _{\beta}\left(S_{1}\right)$, and the $l_{1}$ bit of the received codeword is complemented. These two cases constitute all possibilities since $S_{2}=\left(S_{1}\right)^{2}$. Therefore, decoding failures are impossible.

\section{The Fundamental Iterative Algorithm}

The FIA uses some or all the zeros of $g(x)$ that the BMA does not, to try to increase the decoded distance of the code. Given the same received block $v(x)$ discussed for the BMA, the syndromes for the matrix $\Xi$ can be calculated such that $S_{i}=v\left(\beta^{i}\right)$, where 


$$
\Xi=\left[\begin{array}{cccc}
S_{a} & S_{a+i_{1} c_{1}} & \ldots & S_{a+i_{t^{\prime}} c_{1}} \\
S_{a+c_{2}} & S_{a+i_{1} c_{1}+c_{2}} & \ldots & S_{a+i_{t^{\prime}} c_{1}+c_{2}} \\
& & \ldots & . \\
S_{a+\left(t^{\prime}-1\right) c_{2}} & S_{a+i_{1} c_{1}+\left(t^{\prime}-1\right) c_{2}} & \ldots & S_{a+i_{t^{\prime}} c_{1}+\left(t^{\prime}-1\right) c_{2}}
\end{array}\right]
$$

Note that $t^{\prime}$ is the FIA's number of correctable errors, as opposed to $t$, which is the BMA's number of correctable errors. The variables $a, c_{1}, c_{2}, i_{1}, i_{2}, \ldots, i_{t^{\prime}}$ are code-specific and have permanent values for each code, where $0<i_{1}<\ldots<i_{t^{\prime}}$. Maximizing $t^{\prime}$, these permanent values are deduced by trial and error using the whole set or a subset of the zeros of $g(x)[3]$.

Since $\Xi$ possesses recurrent rows (i.e. rows equally spaced by an index of $c_{2}$ ), the decoder algorithm complexity is $O\left(n^{2}\right)$. With $c_{1}=c_{2}=1$ and $i_{j+1}=i_{j}+1$, for all $j \in 0, \ldots, t^{\prime}-1$, where $i_{0}=0$, the FIA degenerates into the BMA since the entries of $\Xi$ constitute a single set of $2 t$ known consecutive syndromes [3].

Given $\Xi$, if the number of symbol errors in the received codeword is less than or equal to $t^{\prime}$, then the FIA can be used to find the coefficient polynomial $\psi(x)$ and the rank of $\Xi, \lambda$. If the FIA degenerates into the BMA, $\psi(x)=\Lambda(x)$. Otherwise, the relationship of $\psi(x)$ and $\Lambda(x)$ is not as clear. In general,

$$
f(x)=\sum_{j=0}^{\lambda} \psi_{j} \cdot x^{i_{\lambda-j}}
$$

where $i_{0}=0$. Also, 


$$
f(x)=h(x) \cdot \Lambda(1 / x)
$$

where $h(x) \in G F\left(q^{m}\right)[x]$. Therefore, $\psi(x)$ and $\Lambda(x)$ have a relationship that is clouded by $h(x)$, which is codeword-dependent [3].

To initialize the FIA, the discrepancy list is set to zero $(D=[0,0, \ldots, 0])$, the discrepancy row position list is set to zero $(u=[0,0, \ldots, 0])$, the column position is set to $1(s=1)$, the row position is set to $1(r=1)$, and $\psi(x)=1$. The following pseudo code describes the inductive section of the FIA, where $d_{r, s}$ is the discrepancy for the current row and column and $\varphi^{(r)}(x)=1+\sum_{i=1}^{t^{\prime}+1} \Xi_{r, i} \cdot x^{i}[3],[4]$ :

labe1 1:

$$
d_{r, s}=\left[\psi(x) \cdot \varphi^{(r)}(x)\right]_{s}=\sum_{i=0}^{s-1} \psi_{i} \cdot \Xi_{r, s-i}
$$

label 2:

$$
\begin{aligned}
& \text { if }\left(d_{r, s}=0\right) \\
& \{ \\
& \text { if }\left(r=t^{\prime}\right) \\
& \{ \\
& \lambda=s-1 \\
& \text { END } \\
& \text { \} } \\
& \text { else } \\
& r=r+1 \\
& \text { goto label } 1 \\
& \text { \} } \\
& \text { \} } \\
& \text { else }
\end{aligned}
$$




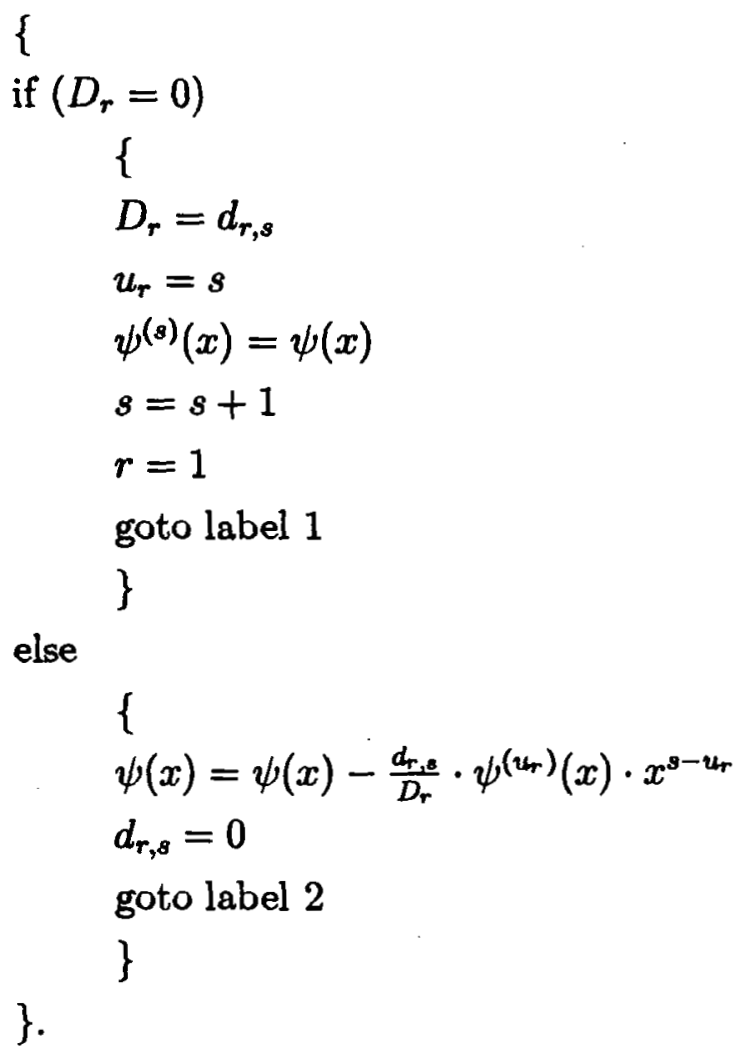

With $\psi(x)$ and $\lambda$ known, the syndrome dependence polynomial $f(x)$ can be constructed. Since $\Lambda(1 / x)$ has zeros of the form $\beta^{e_{l}}$, where $e_{l}$ is the position of the $l^{\text {th }}$ potential error, a Chien search can be used on $f(x)$ to eliminate as many zeros of $h(x)$ as possible. All the zeros found with the Chien search are considered members of the set $U$, where $|U|=\delta$. Using these members, a polynomial similar to $\Lambda(x)$ can be constructed

$$
\Gamma(x)=\prod_{l=1}^{\delta}\left(1-x \cdot U_{l}\right)
$$

Some zeros of $h(x)$ might be used in building $\Gamma(x)$. Otherwise, $\Gamma(x)=\Lambda(x)$, which is 
capable of revealing $t^{\prime}$ or less error locations, as opposed to the BMA's $\Lambda(x)$, which can only reveal $t$ or less error locations [3].

Given the largest set of known consecutive syndromes $\xi$, the Forney Algorithm requires $|\xi| \geq \delta$ or more specifically $|\xi|=\max \{\delta\}$. For all cases where $\Xi$ has entries that form two or more distinct sequences of known consecutive syndromes (i.e. multisequence cases), $|\xi| \leq 2 t^{\prime}-1$. Therefore, if $\delta \geq 2 t^{\prime}$, the number of known consecutive syndromes must be increased. These extra syndromes may or may not be found using the $n$ syndrome linear dependence equations or "patch" equations described by

$$
S_{a+i_{\lambda} c_{1}+j c_{2}}+f_{1} S_{a+i_{\lambda-1} c_{1}+j c_{2}}+\ldots+f_{\lambda} S_{a+j c_{2}}=0
$$

where $j \in 0, \ldots, n-1$. Only $t^{\prime}$ of these equations are necessary, one for each possible non-zero value of $\lambda$. The value of $j$ for each equation can be found by trial and error. If the extra syndromes cannot be found, the FIA will not be able to correct $t^{\prime}$ errors even though $\Xi$ is a $t^{\prime} \times t^{\prime}+1$ matrix of the form described previously [3].

Assuming $|\xi| \geq \delta$, an adapted form of the Forney Algorithm can be used to find the error weights

$$
W_{e_{j}}=-\frac{\Omega\left(\beta^{-e_{j}}\right)}{\Gamma^{\prime}\left(\beta^{-e_{j}}\right)}
$$

where

$$
\begin{aligned}
& \Omega(x)=S(x) \Gamma(x)\left(\bmod x^{|\xi|}\right), \\
& S(x)=\sum_{j=1}^{|\xi|} S_{a+j-1} x^{(j-1)}
\end{aligned}
$$




$$
\Gamma^{\prime}(x)=\sum_{i=1}^{\delta}\left[\sum_{j=1}^{i} 1\right] \Gamma_{i} x^{(i-1)}
$$

Therefore, $e(x)$ is fully specified by

$$
e(x)=\sum_{j=1}^{\delta} W_{e_{j}} \cdot x^{e_{j}}
$$

and,

$$
\begin{aligned}
& c(x)=v(x)-e(x), \\
& i(x)=c(x) / g(x) .
\end{aligned}
$$

For the $(17,9)$ code, $t^{\prime}=2$. The syndrome matrix $\Xi$ is

$$
\Xi=\left[\begin{array}{ccc}
S_{1} & S_{8} & S_{15} \\
S_{2} & S_{9} & S_{16}
\end{array}\right]
$$

where $a=1, c_{1}=1, c_{2}=1, i_{1}=7$, and $i_{2}=14$. Since $\delta \leq 8,|\xi|$ needs to be 8 , so the following patch equations were found by trial-and-error by evaluating the linear dependence equation at $j=0, \ldots, n-1$ :

$$
\begin{aligned}
S_{15} \cdot \psi_{1}+S_{5} & =0, \text { for } \lambda=1 \text { and } j=14 \\
S_{8} \cdot \psi_{2}+S_{15} \cdot \psi_{1}+S_{5} & =0, \text { for } \lambda=2 \text { and } j=7
\end{aligned}
$$

For $j=t^{\prime}, \ldots, n-1$, the equation does not contain syndromes from a particular row in $\Xi$. These syndromes are contained in a particular extension row of $\Xi$. For example, $S_{8}, S_{15}$, and $S_{5}$ comprise the sixth extension row of $\Xi$, which corresponds to $j=7$. 
For $j=14, S_{15}$ and $S_{5}$ comprise the first two elements of the thirteenth extension row. Note that $S_{1}, S_{2}, S_{4}, S_{8}, S_{9}, S_{13}, S_{15}$, and $S_{16}$ are all known. If $S_{3}, S_{5}, S_{6}$, and $S_{7}$, could be found, $|\xi|=8$. Therefore, for each possible value of $\lambda$, the syndrome dependence equation was examined for each value of $j$, until it became an equation with a single-term unknown that was in the conjugate set containing $S_{3}, S_{5}, S_{6}, S_{7}$, $S_{10}, S_{11}, S_{12}$, and $S_{14}$.

The generator polynomial is the same as the that for the BMA, but the following version is multiplied out to show the coefficients in $G F(2)$

$$
g(x)=x^{8}+x^{7}+x^{6}+x^{4}+x^{2}+x+1 .
$$

As an example, assume that the information polynomial is $i(x)=1$ and the error polynomial is $e(x)=x^{8}+1$. Then, the codeword polynomial is $c(x)=x^{8}+x^{7}+$ $x^{6}+x^{4}+x^{2}+x+1$, and the received polynomial is $v(x)=x^{7}+x^{6}+x^{4}+x^{2}+x$. Calculating syndrome $S_{1}$ and using the relationships $S_{2}=\left(S_{1}\right)^{2}, S_{8}=\left(S_{2}\right)^{4}, S_{16}=$ $\left(S_{8}\right)^{2}, S_{15}=\left(S_{16}\right)^{2}$, and $S_{9}=\left(S_{15}\right)^{4}$ gives

$$
\Xi=\left[\begin{array}{ccc}
\alpha^{9} & \alpha^{72} & \alpha^{33} \\
\alpha^{18} & \alpha^{132} & \alpha^{144}
\end{array}\right] .
$$

Now, $\psi(x)$ and $\lambda$ can be found with the FIA:

Initialization:

$$
D=[0,0] ; u=[0,0] ; s=1 ; r=1 ; \psi(x)=1
$$


Loop 1:

$$
\begin{aligned}
& d_{1,1}=\psi_{0} \cdot \Xi_{1,1}=\alpha^{9} \\
& D=\left[\alpha^{9}, 0\right] ; u=[1,0] ; \psi^{(1)}(x)=1 ; s=2 ; r=1
\end{aligned}
$$

Loop 2:

$$
\begin{aligned}
& d_{1,2}=\psi_{0} \cdot \Xi_{1,2}+\psi_{1} \cdot \Xi_{1,1}=\alpha^{72} \\
& \psi(x)=\psi(x)-\frac{d_{1,2}}{D_{1}} \cdot \psi^{(1)}(x) \cdot x=1+\alpha^{63} x ; r=2
\end{aligned}
$$

Loop 3:

$$
\begin{aligned}
& d_{2,2}=\psi_{0} \cdot \Xi_{2,2}+\psi_{1} \cdot \Xi_{2,1}=\alpha^{64} \\
& D=\left[\alpha^{9}, \alpha^{64}\right] ; u=[1,2] ; \psi^{(2)}(x)=1+\alpha^{63} x ; s=3 ; r=1
\end{aligned}
$$

Loop 4:

$$
\begin{aligned}
& d_{1,3}=\psi_{0} \cdot \Xi_{1,3}+\psi_{1} \cdot \Xi_{1,2}+\psi_{2} \cdot \Xi_{1,1}=\alpha^{254} \\
& \psi(x)=\psi(x)-\frac{d_{1,3}}{D_{1}} \cdot \psi^{(1)}(x) \cdot x^{2}=1+\alpha^{63} x+\alpha^{245} x^{2} ; r=2
\end{aligned}
$$

Loop 5:

$$
\begin{aligned}
& d_{2,3}=\psi_{0} \cdot \Xi_{2,3}+\psi_{1} \cdot \Xi_{2,2}+\psi_{2} \cdot \Xi_{2,1}=\alpha^{161} \\
& \psi(x)=\psi(x)-\frac{d_{2,3}}{D_{2}} \cdot \psi^{(2)}(x) \cdot x=1+\alpha^{199} x+\alpha^{75} x^{2} \\
& \lambda=2
\end{aligned}
$$

This $\psi(x)$ and $\lambda$ yield $f(x)=x^{14}+\alpha^{199} x^{7}+\alpha^{75}$. Using a Chien search, the zeros of $f(x)$ are $\alpha^{0.15}$ and $\alpha^{8.15} ;$ therefore, $\Gamma(x)=\alpha^{120} x^{2}+\alpha^{9} x+1$. For this code, $|\xi|=8$. Also, for this example, $\lambda=2$. Therefore, $S_{3}, S_{5}, S_{6}$, and $S_{7}$ must be determined using the $\lambda=2$ patch equation in order to have 8 known consecutive syndromes. All 
four unknown syndromes are in the same conjugate set, so $S_{5}$ can be deduced from the patch equation and then the following equations can be used to find $S_{3}, S_{6}$, and $S_{7}:$

$$
\begin{aligned}
& \left(S_{5}\right)^{4}=S_{3}, \\
& \left(S_{3}\right)^{2}=S_{6}, \\
& \left(S_{6}\right)^{4}=S_{7} .
\end{aligned}
$$

It may be confusing why $|\xi|=\max \{\delta\}=8$. This value is determined by constructing a FIA decoder in software. This software is used to decode all possible error patterns containing $t^{\prime}$ or less errors starting with $|\xi|=2 t^{\prime}$ and increasing $|\xi|$ after each attempted verification until the decoder corrects all of these error patterns. This process finds the minimum value of $|\xi|$ required to properly decode $t^{\prime}$ or less errors.

With $S_{1}$ through $S_{8}$ and $\Gamma(x)$ known, the Forney algorithm can be used to identify which zeros of $f(x)$ of the form $\beta^{x}$ correspond to actual error locations. For this example, both zeros are identified, but there are many cases where the identified zeros are a subset of the zeros found with the Chien search. With the two zeros identified, $e(x)=x^{8}+1$, which is the correct error pattern.

The relation between the FIA and the BMA is not easily seen; however, there is one. Starting with column one, the FIA uses a truncated BMA; it starts out like the BMA but stops if a discrepancy, $d_{r, s}$, is non-zero. This discrepancy and its column number are stored as $D_{r}$ and $u_{r}$ along with the current approximation of the coefficient 
polynomial, $\psi(x)$, which is stored as $\psi^{(s)}(x)$. For the other columns, the initialization is dependent upon all previous columns; the procedure is similar to the procedure for the first column, except when a non-zero discrepancy is encountered, the truncated BMA will stop only if no discrepancy has been recorded for that particular row, in essence, if $D_{r}=0$. If $d_{r, s} \neq 0$ and $D_{r} \neq 0, \psi(x)$ is updated. The algorithm continues this pattern for each column until it is able to perform the full BMA on a column. It is possible that the full BMA might be performed on the first column, but this would indicate that no errors could be found. 


\section{CHAPTER III}

\section{PERFORMANCE COMPARISONS}

Since theoretical performance measures of $\mathrm{BCH}$ codes are inexact, simulation was necessary to empirically measure performance. Therefore, a baseband digital communication system using BMA and FIA decoders was programmed on a computer.

This simulated system was used to approximate the bit error rate (BER) at the decoder output versus SNR curve for both the BMA and the FIA. This curve is important since it gives insight into the system coding gain, which is an important performance indicator.

Let $p_{u}(h)$ be the BER for the uncoded system operating at the SNR $h$, and let $p_{c}(h)$ be defined in a similar way for the coded system. Then, the coding gain is defined as

$$
g(\rho)=p_{c}^{-1}(\rho)-p_{u}^{-1}(\rho)
$$

where $\rho$ is the BER and all SNR's are in dB. Therefore, the coding gain at a given BER is the difference in SNR of the uncoded and coded systems when each are operating at a specific BER [11]. 


\section{The Simulated System}

This statistical computer simulation entails:

1. Generating an $X$ bit maximum entropy encoder input sequence,

2. Encoding in $k$ symbol blocks at a $k / n$ input/output rate, which involves constructing an $n$-length channel codeword from a $k$-length source codeword, where each component is a binary symbol,

3. Generating an effective channel error sequence of length $X n / k$ such that the errors are exponentially spaced. The probability of an error is approximately the BER of the coded channel, and the number of decoder bit errors $\chi$ is large (greater than 100) [2],

4. Adding the error sequence to the encoded sequence bit by bit in $G F(2)$,

5. Decoding in $n$ symbol blocks at an $n / k$ input/output rate.

It is assumed that source encoding, if necessary, has been applied so that the binary symbols at the encoder input are independent and equally likely. This input can be modeled by a pseudo-noise (PN) sequence. A PN sequence is periodic with a maximum period of $N=2^{r}-1$, where $r$ is the number of stages in the generating shift register [10]. In many cases, tap weights can be selected so that the maximum period is achieved, resulting in a maximal-length sequence [10]. Each maximal-length sequence has at least three non-zero taps. Those sequences with only three taps are the most time efficient, since the generation speed increases as the number of non-zero taps decrease. The periodic autocorrelation $R_{p}(u)$ is defined as $\left(N_{0}-N_{1}\right) /\left(N_{0}+N_{1}\right)$, 
where $p$ is a sequence with period $N=N_{0}+N_{1}, s$ is the modulo-2 addition of $p$ and its $u$ position cyclic shift, $N_{0}$ is the number of 0 's in $s$, and $N_{1}$ is the number of 1's in $s$. Any modulo-2 addition of a maximal-length sequence with its $u$ position cyclic shift is the $u^{\prime}$ position cyclic shift of the maximal-length sequence. Also, the number of 1 's in a maximal-length sequence is one greater than the number of 0 's; therefore, the periodic autocorrelation function of any maximal-length sequence is [10]

$$
R_{p}(u)=\left\{\begin{array}{rll}
1.0 & \text { if } & u=l N \\
-\frac{1}{N} & \text { if } & u \neq l N
\end{array}\right.
$$

where $l \in I$. As $r$ approaches $\infty, N$ approaches $\infty$, and the correlation dwindles to 0.

For a simulation, $N=2^{x}-1$, where $x=\left\lceil\log _{2} X\right\rceil$ and $X$ is the number of input bits required by the encoder for an entire simulation. $X$ is difficult to determine, since the calculation of $X$ is based on the coded BER $\rho$

$$
X=\frac{(\chi)(k)}{\rho n},
$$

where $\chi$ is the number of bit errors at the output of the decoder. The BER at the decoder output is actually a random variable (RV), which can have a multimodal distribution if $X$ is not large enough. The goal is to choose $X$ so that $\chi$ is large (greater than 100) [2].

The probability of a bit error at the output of the raw channel when an encoder and decoder are present is the BER of the coded channel $\psi_{0}$. If $\psi_{0} \geq 10^{-2}$, the effective 
channel error sequence can be constructed by thresholding successive outputs of a uniform pseudo random number with $\psi_{0}$. If the output is less than or equal to $\psi_{0}$, a 1 is declared; otherwise, a 0 is declared. If $\psi_{0}<10^{-2}$, the following approach is used. The location of the first error is calculated by [6]

$$
\Delta s=-\frac{1}{\psi_{o}} \ln (u),
$$

where $\Delta s$ is the number of zero sequence elements until an error occurs and $u$ is a uniform pseudo random number. Subsequent error locations can be determined by using the same equation where $\Delta s$ is the number of zero sequence elements until the next error and $u$ is a new uniform pseudo random number [6].

\section{The Results}

For the $(17,9) \mathrm{BCH}$ code with $g(x)=m_{1}(x)$ and a minimum distance $d=5$, the exploited distance is 4 for the BMA and 5 for the FIA, yielding $t=1$ and $t^{\prime}=2$, respectively. Figure 3.1 shows the corresponding theoretical performance curves of error probability versus $E_{b} / N_{o}$. The theoretical BER at the output of the decoder is given by [7]

$$
P(\text { bit error at decoder output })=\sum_{j=t+1}^{n}\left[\frac{j}{n} \cdot\left(\begin{array}{l}
n \\
j
\end{array}\right) \cdot p^{j} \cdot(1-p)^{n-j}\right],
$$

where 


$$
\begin{gathered}
P(j \text { errors in codeword })=\left(\begin{array}{c}
n \\
j
\end{array}\right) \cdot p^{j} \cdot(1-p)^{n-j}, \\
P(\text { bit error } \mid j \text { errors in a codeword })=\frac{j}{n}
\end{gathered}
$$

Note that for the FIA the summation actually ranges from $t^{\prime}+1$ to $n$. For the theoretical plots, the FIA coding gain is positive for all SNR's above $3.2 \mathrm{~dB}$, and the BMA coding gain is positive for all SNR's above $11 \mathrm{~dB}$. Also, the FIA net coding gain is $1.5 \mathrm{~dB}$ and $1.8 \mathrm{~dB}$ for system BER's $10^{-5}$ and $10^{-8}$, respectively, whereas the BMA net coding gain is small for both these system BER's.

To validate the simulation, decoding errors (those errors that give rise to undetected word errors) were disabled by giving the decoder knowledge of the transmitted codewords. Figures 3.2 and 3.3 compare the simulation results and the theoretical curves for the BMA and FIA, respectively. Each simulation curve coincides with its corresponding theoretical curve. Therefore, both simulation models are considered valid.

Next, both simulations were performed without restriction. Figures 3.4 and 3.5 compare the results with the theoretical curves and the Torrieri Bounds [8]. The Torrieri Bounds assume that the number of bit errors in a codeword has a binomial distribution (independent bit errors). If a codeword has $t$ errors or less for the BMA or $t^{\prime}$ errors or less for the FIA, the codeword is assumed to be fully correctable; otherwise, 
the decoder is assumed to add (worst case) $t$ errors for the BMA or $t^{\prime}$ errors for the FIA to the codeword for the upperbound calculation, and subtract (best case) $t$ errors for the BMA or $t^{\prime}$ errors for the FIA for the lowerbound calculation. For both the BMA and FIA simulations, the simulation curves were just above the theoretical curves, indicating that in the event of a decoding error, the decoder added errors to the received codeword slightly more often than it subtracted errors [8]. Figure 3.6 shows the simulation performance curves for both the BMA and the FIA. At a BER of $10^{-5}$, the BMA coding gain was $-.25 \mathrm{~dB}$, and the FIA coding gain was $1.4 \mathrm{~dB}$. The $10^{-8}$ BER coding gains are not available since the simulation was not performed for high SNR due to the exponential growth in required execution time. 


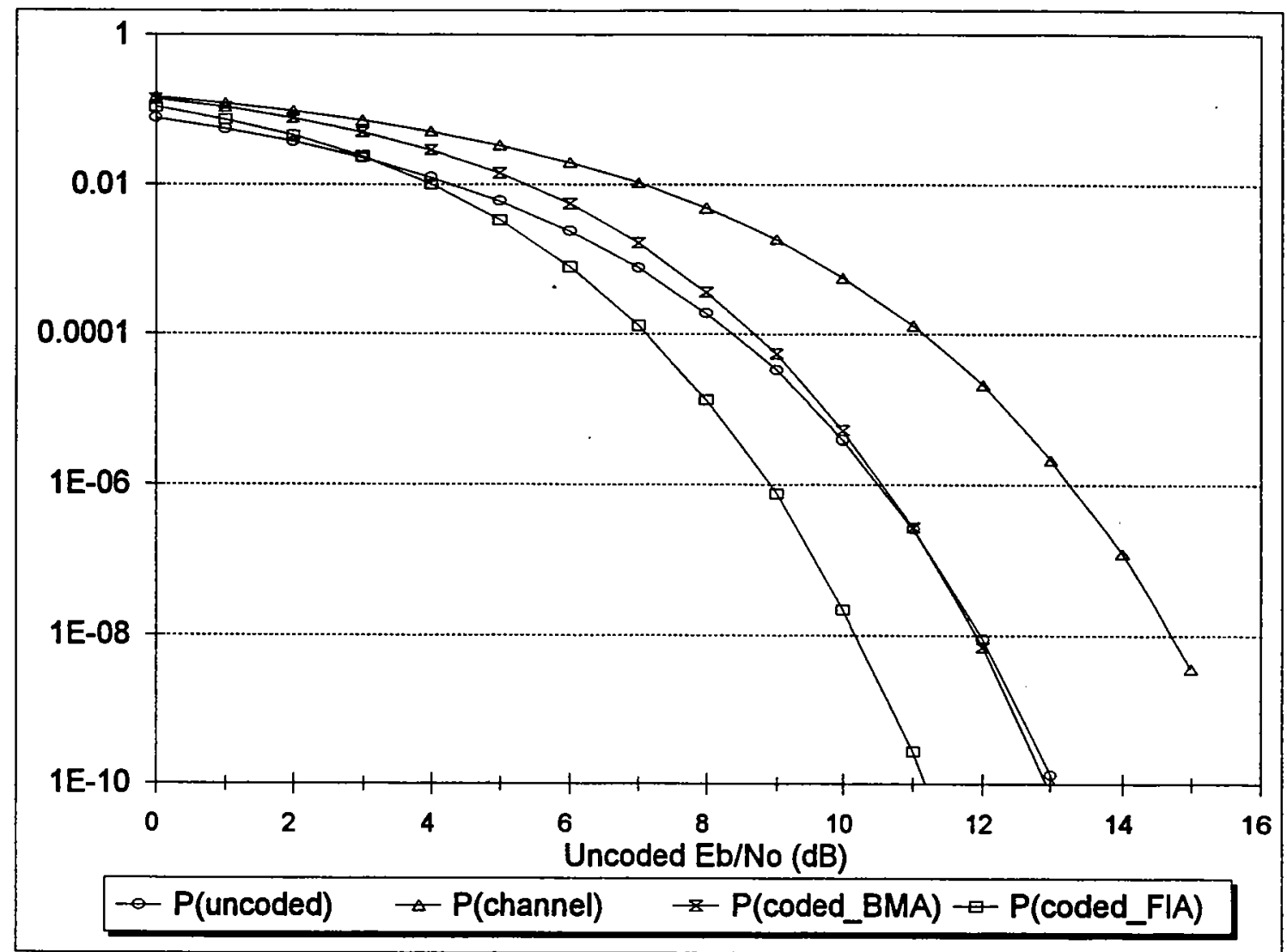

Figure 3.1. THEORETICAL PERFORMANCE CURVES FOR $(17,9)$ BCH CODE 


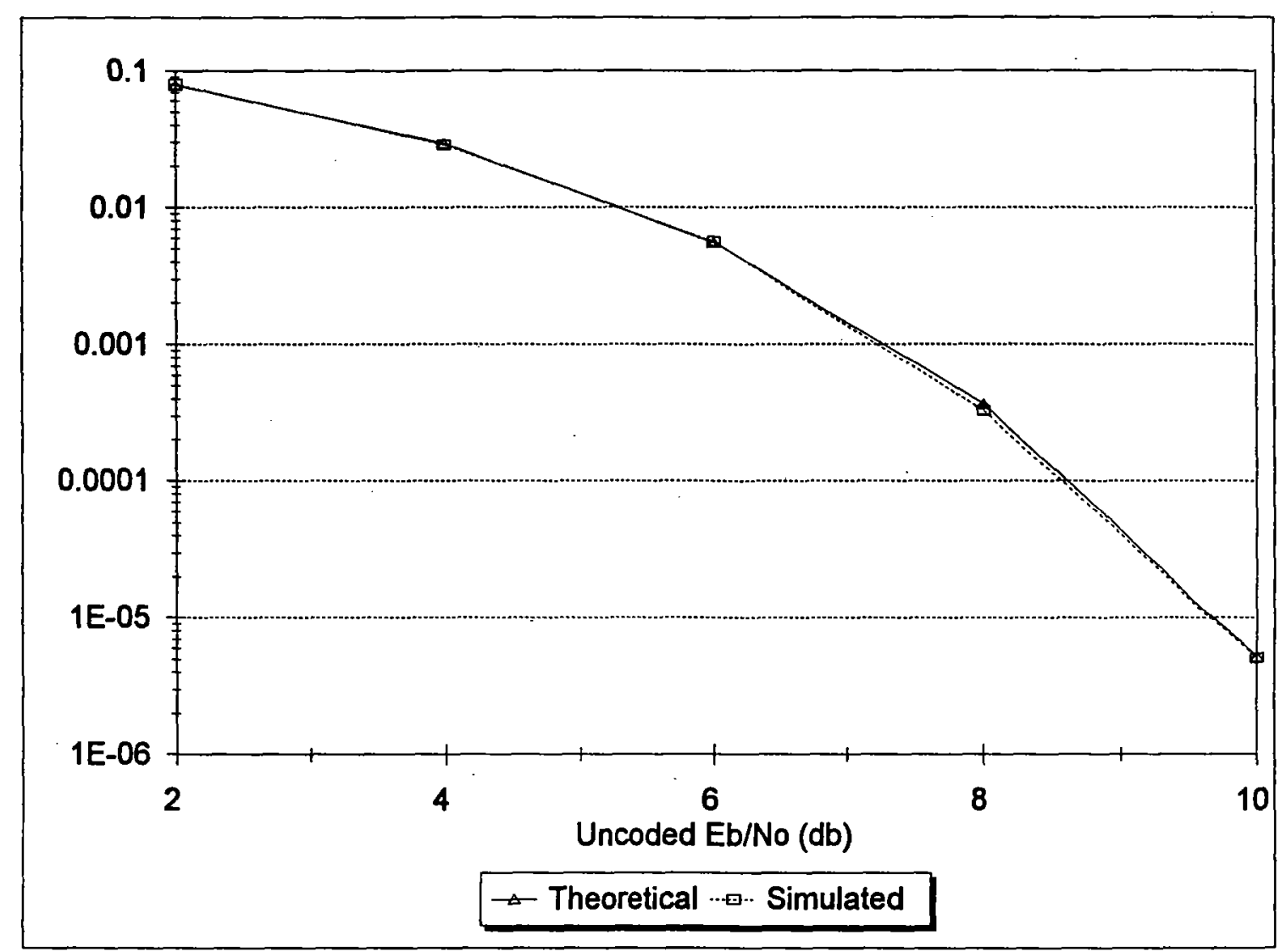

Figure 3.2. BMA STATISTICAL TEST WITH DECODING ERRORS DISABLED FOR $(17,9)$ BCH CODE 


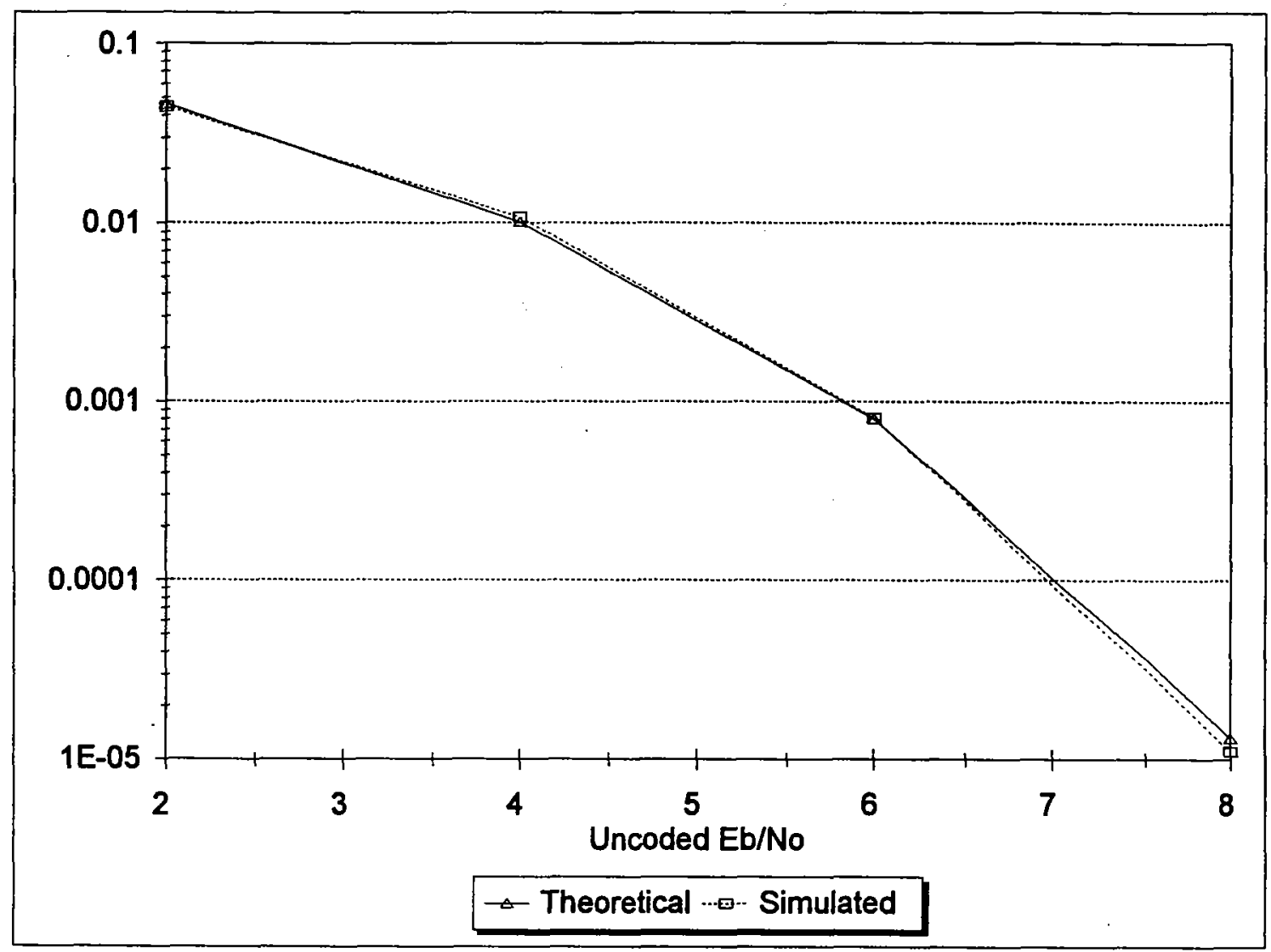

Figure 3.3. FIA STATISTICAL TEST WITH DECODING ERRORS DISABLED FOR $(17,9)$ BCH CODE 


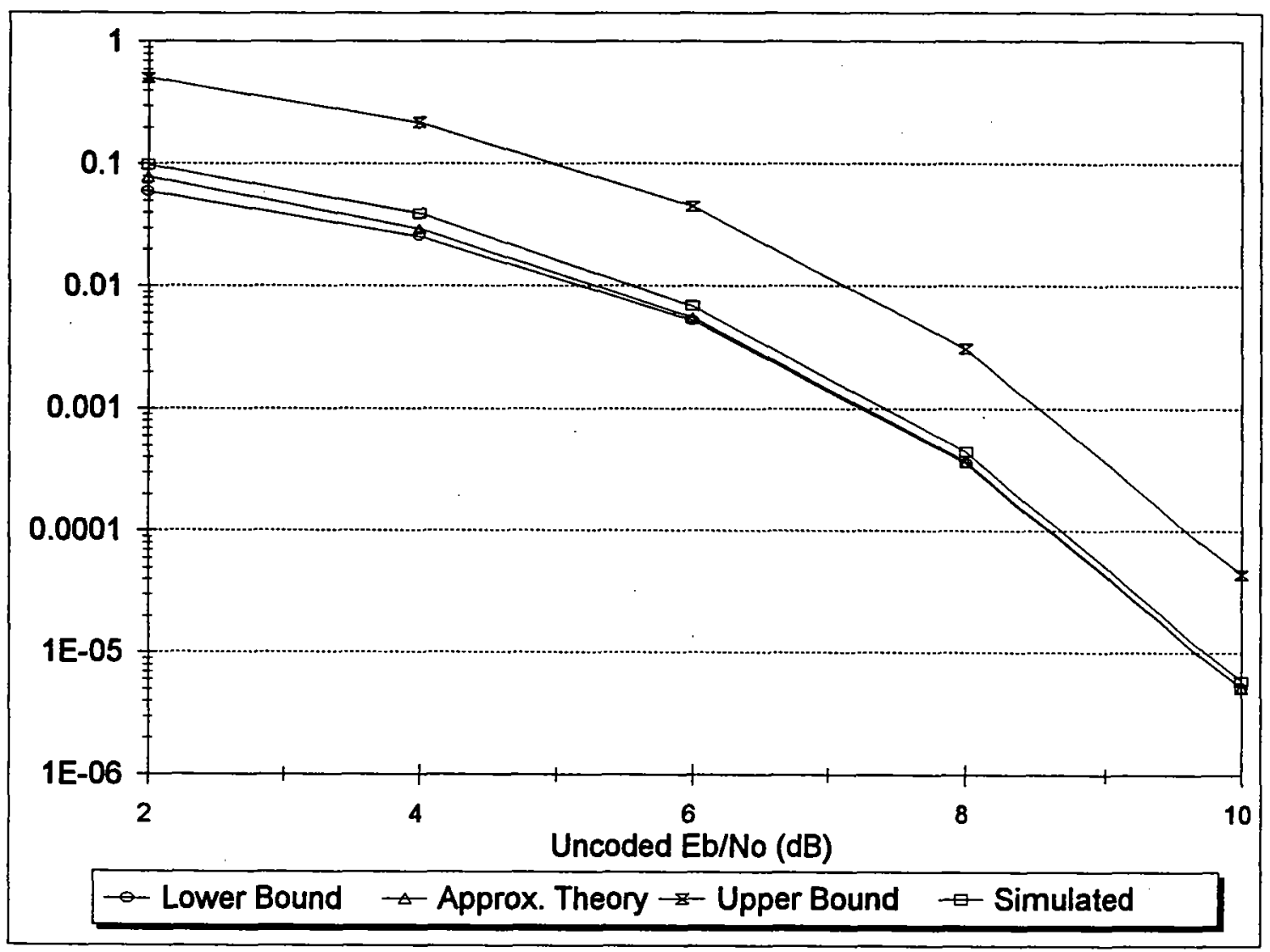

Figure 3.4. BMA STATISTICAL TEST WITH NO RESTRICTIONS FOR $(17,9)$ BCH CODE 


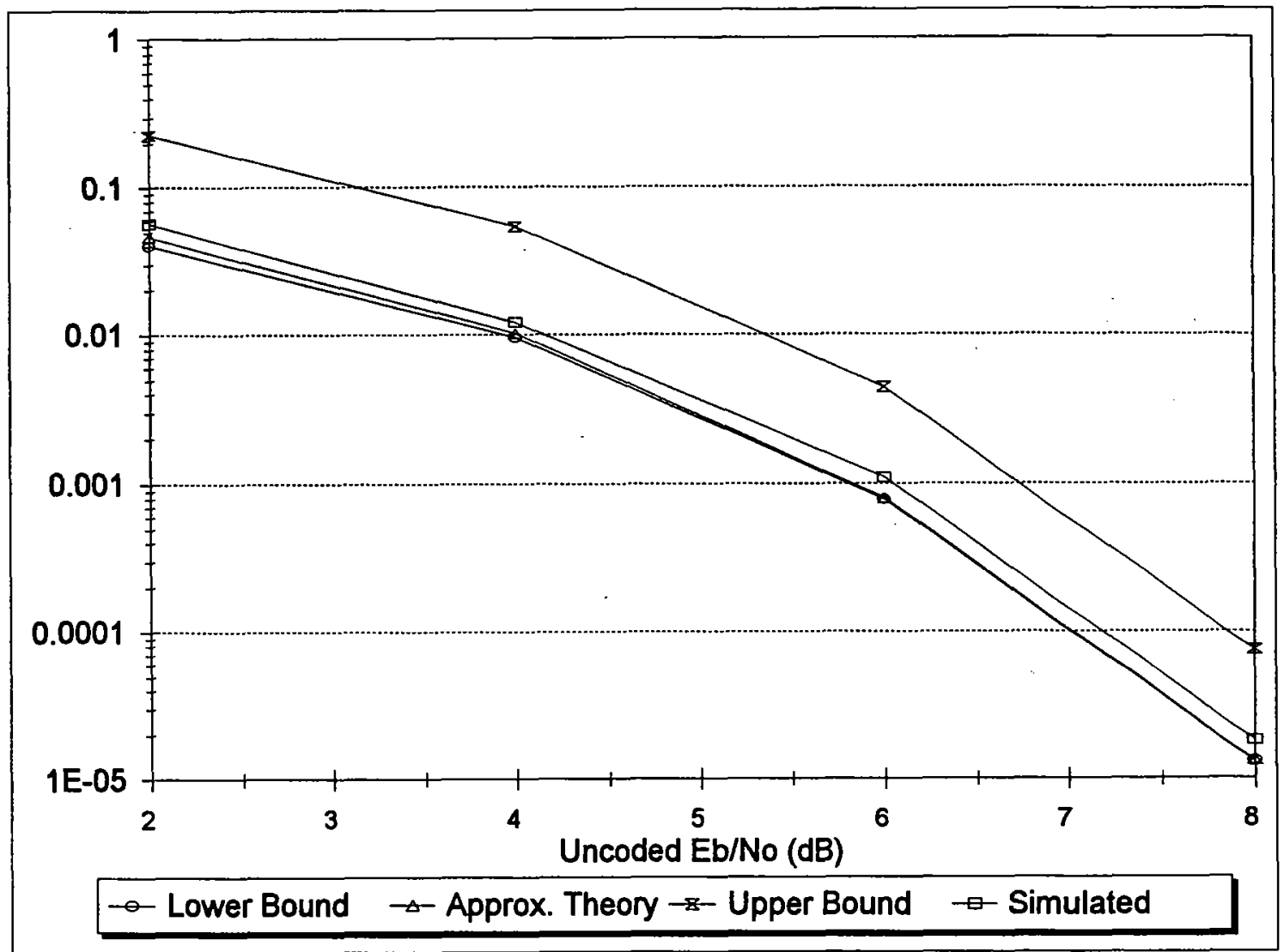

Figure 3.5. FIA STATISTICAL TEST WITH NO RESTRICTIONS FOR $(17,9)$ BCH CODE 


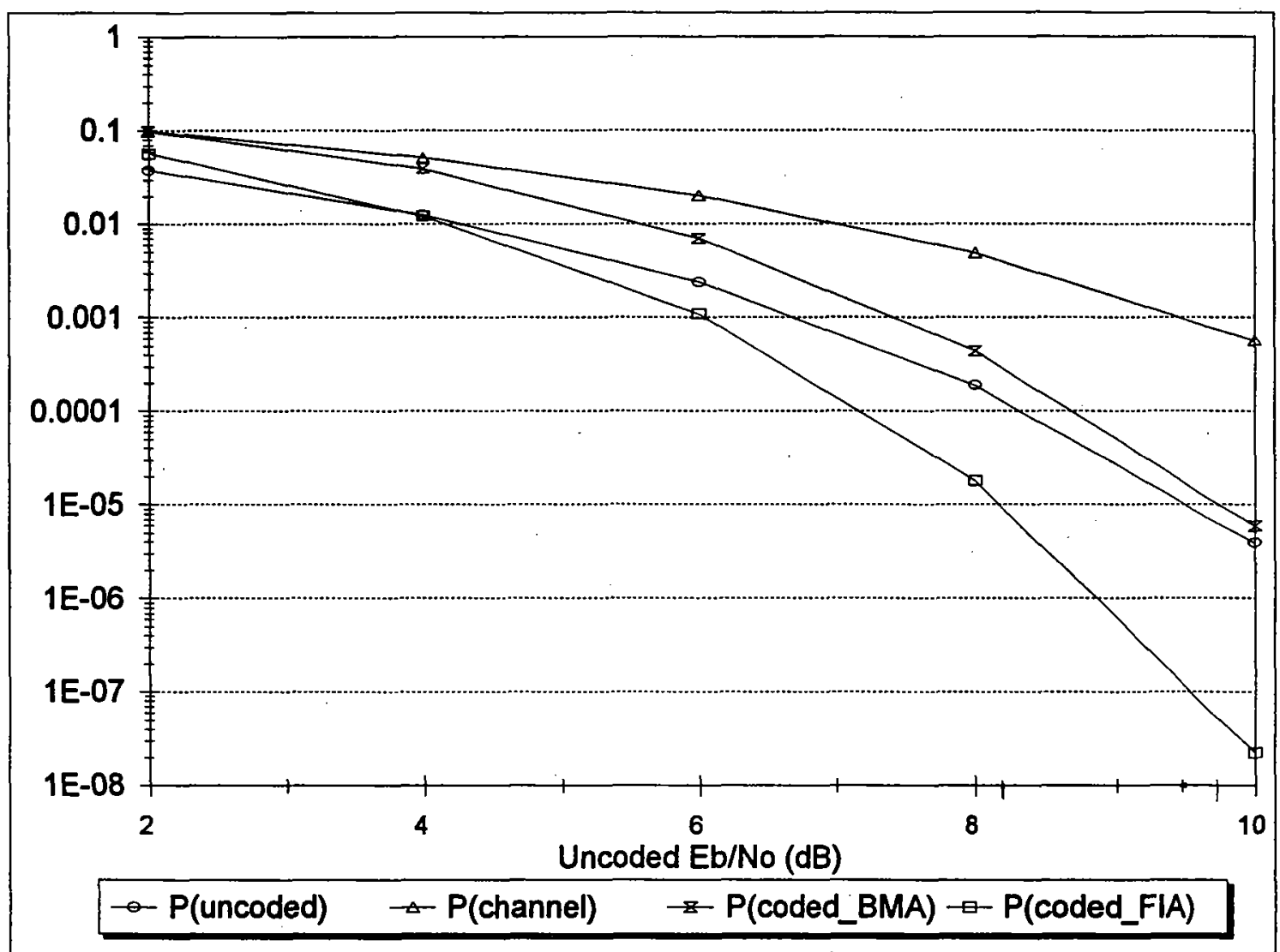

Figure 3.6. SIMULATION PERFORMANCE CURVES FOR $(17,9)$ BCH CODE 


\section{CHAPTER IV}

\section{HARDWARE COMPARISONS}

Two areas of comparison are examined in this chapter. First, the critical path is identified. The critical path is the hardware complexity of the algorithm bottleneck; it is measured with respect to the processing of one received bit. Second, hardware complexity of the entire algorithm is identified. Hardware complexity is similar to chip real-estate analysis but is viewed at a higher level. For this chapter, the complexity will be measured by the number of GF additions, GF multiplications, GF multiplicative inverses, arithmetic additions, arithmetic multiplications, comparisons, temporary memory slots, and permanent memory slots.

\section{Critical Path for the BMA}

Figure 4.1 shows the high-level hardware layout for the entire decoder. This layout calculates the syndrome $S_{1}$ using Horner's rule [1]. The latency of this syndrome calculation is the time required to perform $n-1$ GF multiplies and $n$ GF additions. The maximum latency of the rest of the decoder is the time require to perform $n-1$ GF multiplies, $1 \mathrm{GF}$ addition, $1 \mathrm{GF}$ multiplicative inverse, $n-1$ arithmetic additions, and $2 n$ comparisons. The calculation of syndromes is then the bottleneck, since $n-1 \mathrm{GF}$ additions will most likely require more time than $1 \mathrm{GF}$ multiplicative 
inverse, $n-1$ arithmetic additions, and $2 n$ comparisons. Therefore, the critical path is $1 \mathrm{GF}$ addition, $1 \mathrm{GF}$ multiplication, and 1 memory element.

\section{Critical Path for the FIA}

Figure 4.2 shows the high-level hardware layout for the entire decoder. This layout also calculates the syndrome $S_{1}$ using Horner's rule [1]. The latency of the calculation and storage of syndromes is the time required to perform $(n+6) \mathrm{GF}$ multiplies, $n$ GF additions, plus RAM loading. The two syndrome RAM's must remain unchanged until the calculation of $\Omega(x)$, in the Forney Algorithm, is completed. In a worst case scenario, this storage time is approximately the time required to perform 6 - $n$ GF multiplies, 6 - $n$ GF additions, 3 GF reciprocals, 3 multiplies, $n$ additions, and $n$ compares, which is much longer than the latency time for the calculation and storage of syndromes, assuming that the time to load the two RAM's is relatively small. Therefore, the critical path lies between loading the RAM and completing the calculation of $\Omega(x)$. Since the critical path is measured with respect to the processing one received bit, the FIA has no obvious critical path, since the bottleneck cannot be dissected in this way.

As a side note, RAM 1 has six bytes of memory ( 8 bits/memory element) with a three bit addressing scheme, and RAM 2 has eight bytes of memory with a three bit addressing scheme. Both RAM's can be concatenated into one 14 byte RAM with a four bit addressing scheme, sacrificing access time. 


\section{Complexity Comparisons}

Table 4.1 compares the hardware complexity of the two algorithms. Since the GF multiply is the most complex of all the operations, assuming polynomial representation for the field elements, the FIA is roughly 20 times more complex than the BMA.

Table 4.1. HARDWARE COMPLEXITY COMPARISON

\begin{tabular}{|l|r|r|r|r|l|l|}
\hline Algorithm & GF + & GF $x$ & GF Reciprocal & + & $\times$ & Comparison \\
\hline \hline BMA & 2 & 2 & 1 & 1 & 0 & 3 \\
\hline FIA & 34 & 42 & 3 & 5 & 1 & 10 \\
\hline
\end{tabular}

\begin{tabular}{|l|r|r|}
\hline Algorithm & Temporary Memory & Permanent Memory \\
\hline \hline BMA & 37 & 0 \\
\hline FIA & 128 & 14 \\
\hline
\end{tabular}



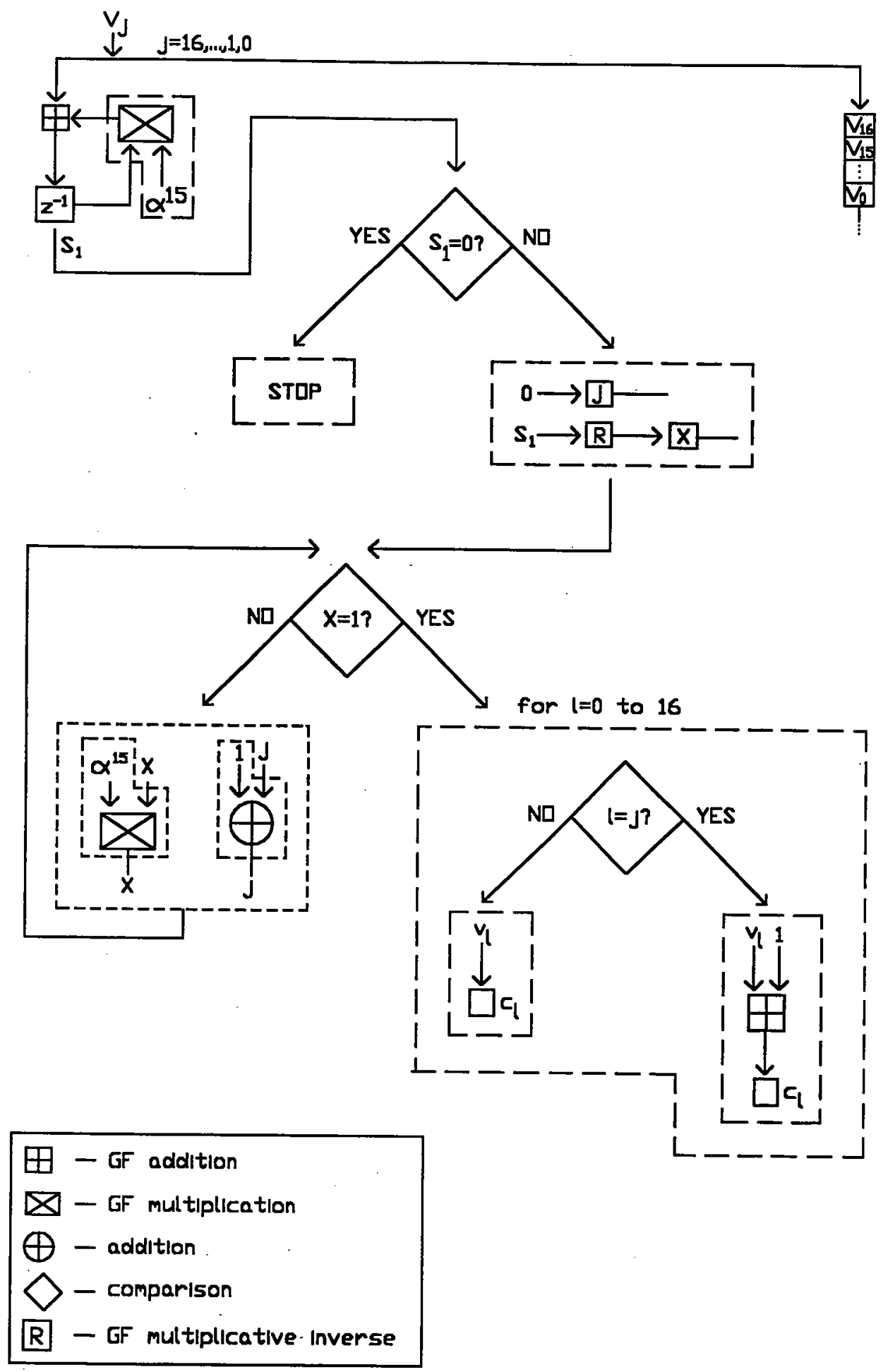

Figure 4.1. BMA HARDWARE LAYOUT FOR A $(17,9)$ BCH DECODER 


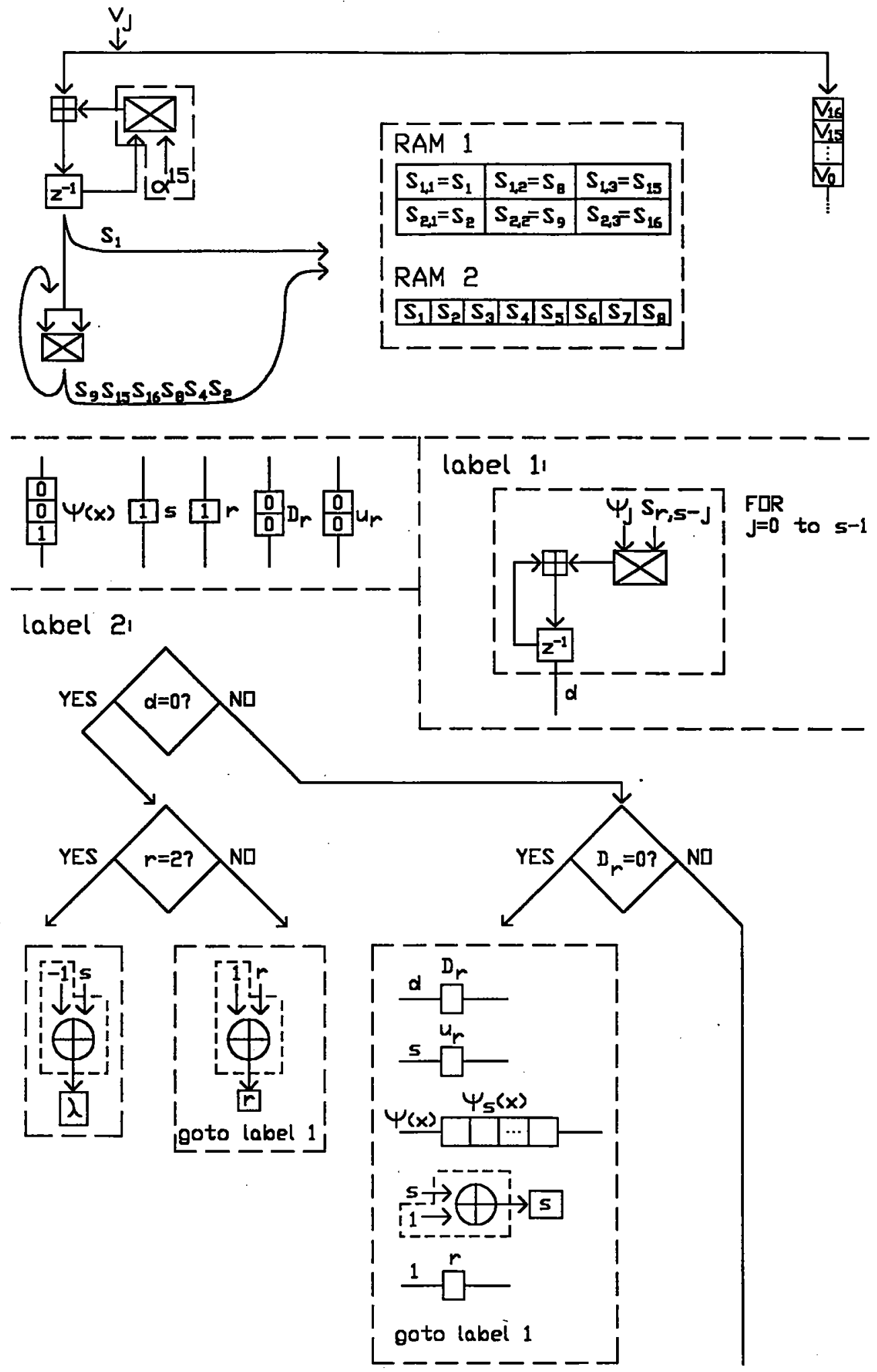

Figure 4.2. FIA HARDWARE LAYOUT FOR A $(17,9)$ BCH DECODER 
FIGURE 4.2. (continued)

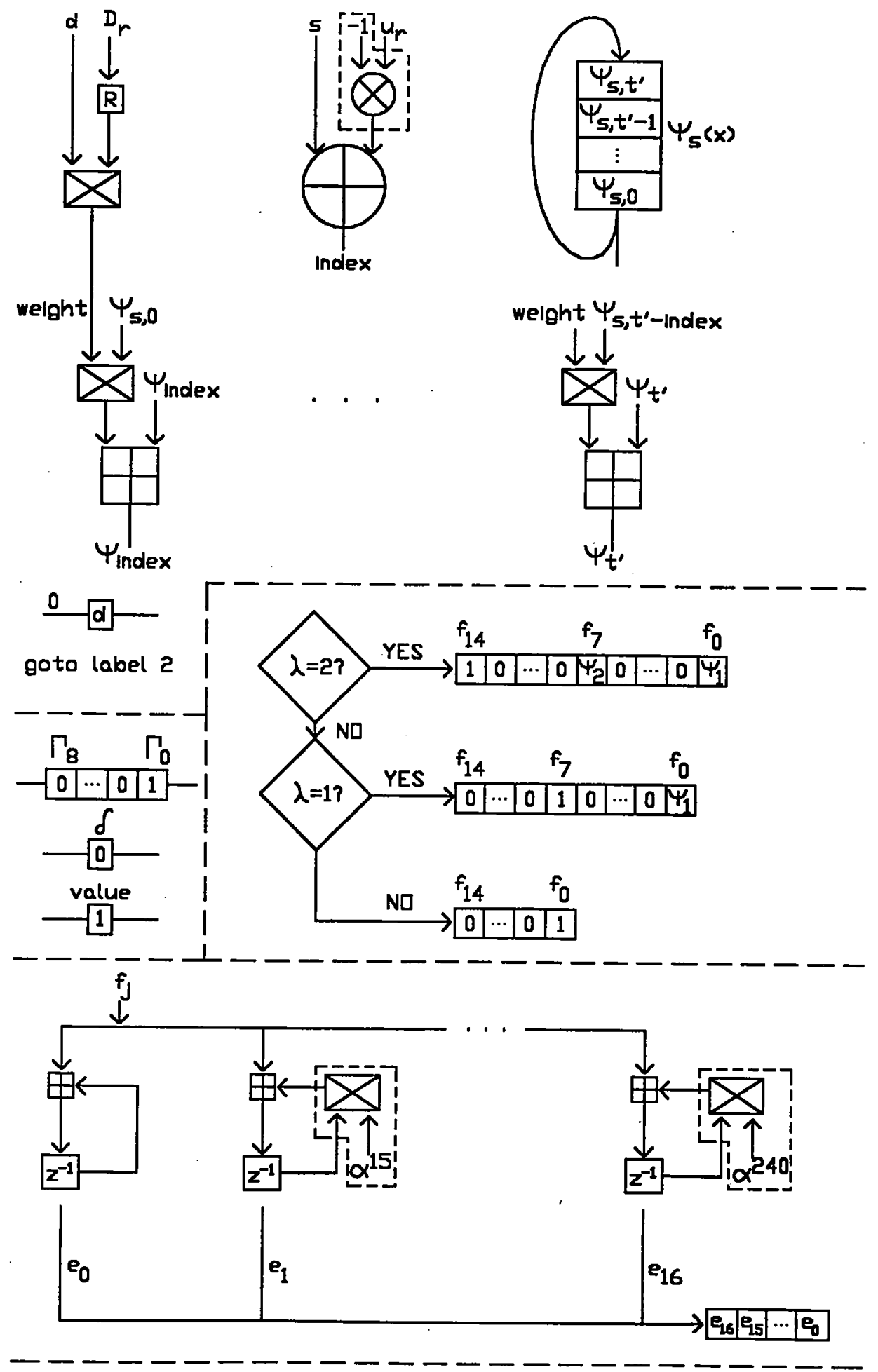


FIGURE 4.2. (continued)

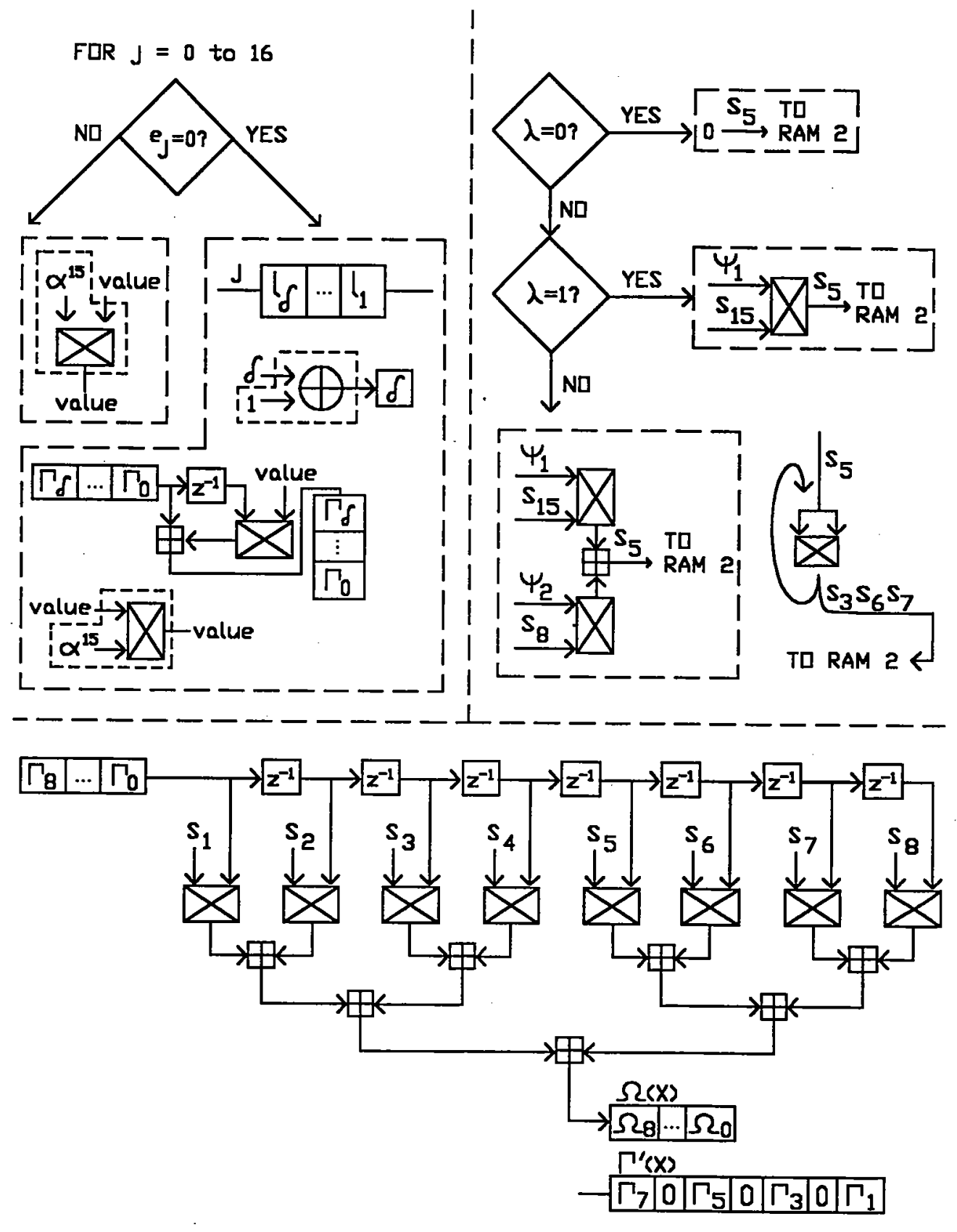


FIGURE 4.2. (continued)
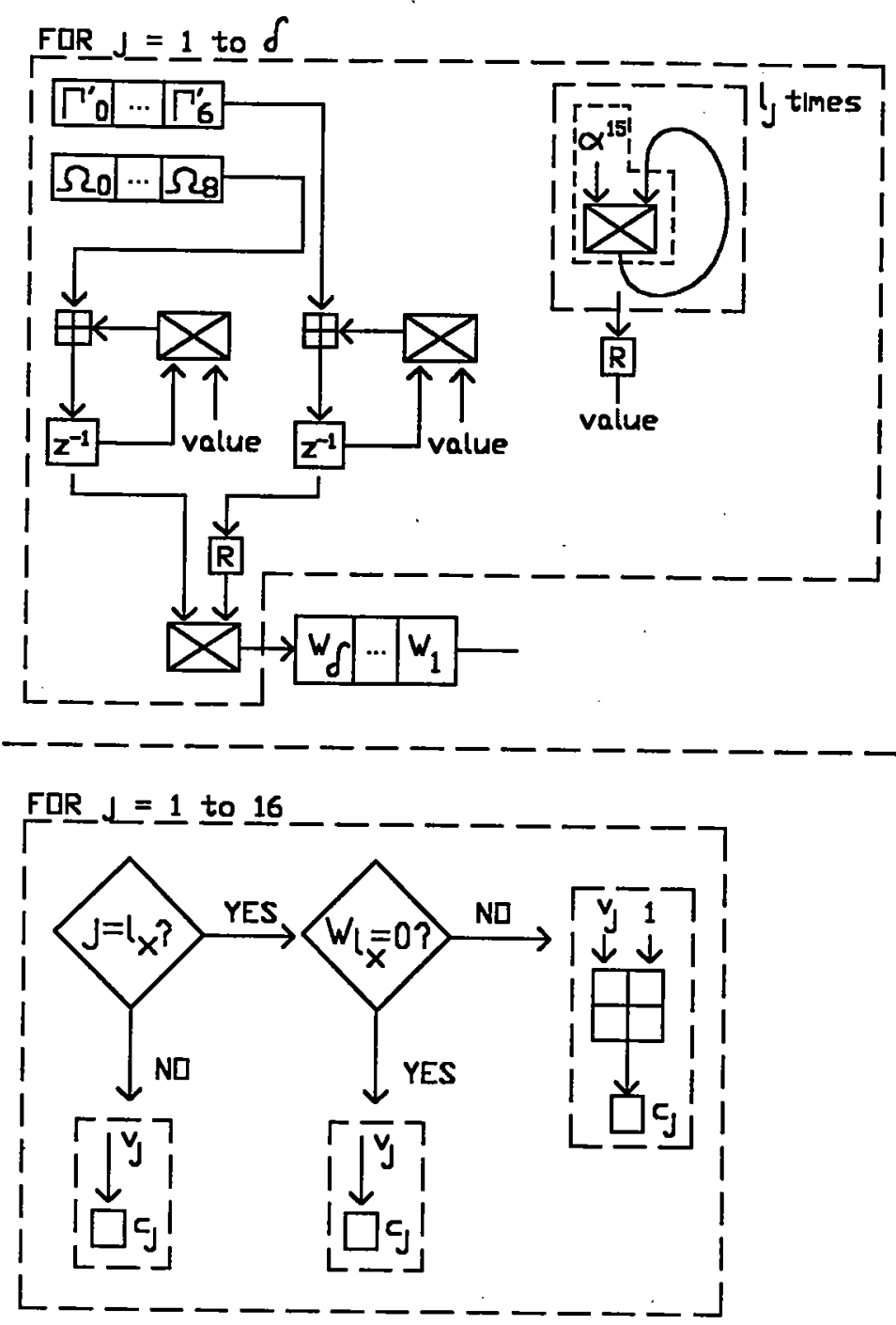

$$
\begin{aligned}
& \boxplus \text { - GF addition } \\
& \bigotimes \text { - GF multiplication } \\
& \oplus \text { - addition } \\
& \otimes \text { - multiplication } \\
& \nabla \text { - comparison } \\
& R \text { - GF multiplicative inverse }
\end{aligned}
$$




\section{CHAPTER V \\ SUMMARY AND CONCLUSIONS}

The BMA uses only the designed distance of a $\mathrm{BCH}$ code, which is often smaller than the true minimum distance [1]; however, the FIA can be used to exploit some or all of the unused distance, in many cases increasing the number of correctable errors [3]. Although the number of correctable errors is an important performance indicator, a more complete indicator is the performance gain/hardware complexity ratio. At a BER of $10^{-5}$, the performance gains are $-.25 \mathrm{~dB}$ and $1.4 \mathrm{~dB}$ for the BMA and FIA, respectively; however, the hardware for the FIA decoder is roughly 20 times more complex than the hardware for the BMA. Also, the critical path for the BMA is $1 \mathrm{GF}$ addition, $1 \mathrm{GF}$ multiplication, and 1 memory element, whereas the FIA is so complex that the critical path not easily identified. 


\section{CHAPTER VI \\ FUTURE DIRECTIONS}

Since the comparison of the BMA and FIA is code-specific, other codes with higher designed distances might be analyzed to give a more balanced comparison. Also, the Extended Fundamental Iterative Algorithm (EFIA) might be analyzed for net coding gain and hardware complexity [4]. 


\section{BIBLIOGRAPHY}

[1] Richard E. Blahut, Theory and Practice of Error Control Codes, Addison Wesley, 1984.

[2] William J. Ebel and Frank M. Ingels, "Confidence intervals for simulations using Reed-Solomon codes," Milcom, 1993.

[3] Gui-Liang Feng and Kenneth K. Tzeng, "Decoding cyclic and BCH codes up to actual minimum distance using nonrecurrent syndrome dependence relations," IEEE Trans. Information Theory, vol. 37, no. 6, pp. 17161722., Nov. 1991.

[4] Gui-Liang Feng and Kenneth K. Tzeng, "A new procedure for decoding cyclic and $\mathrm{BCH}$ codes up to actual minimum distance," IEEE Trans. Information Theory, vol. 40, no. 5, pp. 1364-1374., Sept. 1994.

[5] M. Jeruchim, "Techniques for estimating the bit error rate in the simulation of digital communication systems," IEEE JSAC, vol. SAC-2, pp. 153-171, Jan. 1984.

[6] Athanasios Papoulis, Probability, Random Variables, and Stochastic Processes, McGraw-Hill, 1991.

[7] Bernard Sklar, Digital Communications Fundamentals and Applications, Prentice Hall, 1988.

[8] D. J. Torrieri, "The Information-Bit Error Rate for Block Codes", IEEE Trans. on Communications, vol. Com-32, no. 4, April 1984.

[9] W. H. Tranter and K. L. Kosbar, "Simulation of communication systems," IEEE Communications Mag., pp. 26-35, July 1994.

[10] R. E. Ziemer and R. L. Peterson, Digital Communications and Spread Spectrum Systems, Macmillan, 1985.

[11] R. E. Ziemer and W. H. Tranter, Principles of Communications, Houghton Mifflin, 1990. 


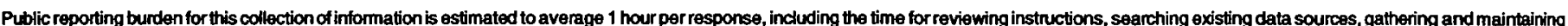

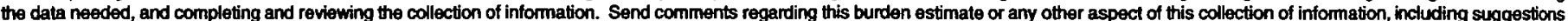

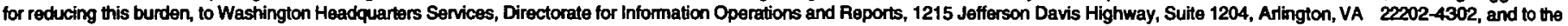
Office of Management and Budget, Paperwork Reduction Project (0704-0188), Washington, DC 20503.

\begin{tabular}{|l|l|l}
\hline 1. AGENCY USE ONLY (Leave blank) & $\begin{array}{l}\text { 2. } \\
\begin{array}{l}\text { REPORT DATE } \\
\text { August } 1996\end{array}\end{array}$ & $\begin{array}{l}\text { 3. } \\
\text { REPORT TYPE AND DATES COVERED } \\
\text { Final report }\end{array}$
\end{tabular}

4. TITLE AND SUBTTILE

A Hardware Analysis of the Fundamental Iterative Algorithm for Decoding A $(17,9)$ Binary $\mathrm{BCH}$ Code
5. FUNDING NUMBERS

6. AUTHOR(S)

Roy L. Campbell, Jr.

7. PERFORMING ORGANIZATION NAME(S) AND ADDRESS(ES)

U.S. Army Engineer Waterways Experiment Station

3909 Halls Ferry Road

Vicksburg, MS 39180-6199

9. SPONSORINGMONITORING AGENCY NAME(S) AND ADDRESS(ES)

U.S. Army Corps of Engineers

Washington, DC 20314-1000
8. PERFORMING ORGANIZATION REPORT NUMBER

Technical Report ITL-96-7 AGENCY REPORT NUMBER

\section{SUPPLEMENTARY NOTES}

Available from National Technical Information Service, 5285 Port Royal Road, Springfield, VA 22161.

12a. DISTRIBUTIONAVAILABILTY STATEMENT

Approved for public release; distribution is unlimited.

12b. DISTRIBUTION CODE

13. ABSTRACT (Maximum 200 words)

The Berlekamp-Massey Algorithm (BMA) is commonly used in BCH decoding, but the Fundamental Iterative Algorithm (FIA) can, in many instances, correct more errors. The trade-off lies in hardware complexity. The BMA has concise stages that do not require addressable memory, whereas the FIA struggles with memory management and complex stages. For the $(17,9) \mathrm{BCH}$ code over $\mathrm{GF}(256)$ with $g(x)=m_{l}(x)$, the FIA can correct up to two errors, but the BMA can correct only one error. Also, for a BER of $10^{-5}$, the coding gains for the BMA and FIA are $-.25 \mathrm{~dB}$ and $1.4 \mathrm{~dB}$, respectively, which makes the FIA seem to be the better algorithm. The main drawback is the FIA is approximately 20 times more complex than the BMA. Also, the FIA does not have a critical path that is easily identified.

14. SUBJECT TERMS

Berlekamp-Massey Algorithm

Fundamental Iterative Algorithm
15. NUMBER OF PAGES

44

16. PRICE CODE
17. SECURITY CLASSIFICATION OF REPORT

UNCLASSIFIED
18. SECURITY CLASSIFICATION OF THIS PAGE UNCLASSIFIED 